ArtefaCToS. Revista de estudios de la ciencia y la tecnología

eISSN: $1989-3612$

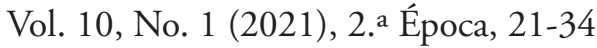

DOI: https://doi.org/10.14201/art20211012134

\title{
Cognición distribuida: entre lo individual y lo social
}

\author{
Distributed Cognition: Between the Individual and the Social
}

\author{
Francesco CONSIGLIO*; Fernando MARTÍNEZ MANRIQUE ${ }^{* *}$ \\ *Universidad de Granada, España \\ consiglio@ugr.es \\ (D) https://orcid.org/0000-0003-0439-8362 \\ **Universidad de Granada, España \\ fmmanriq@ugr.es \\ D https://orcid.org/0000-0002-8671-4850
}

Recibido: 31/01/2021. Revisado: 11/03/2021. Aceptado: 17/03/2021

\section{Resumen}

Ronald Giere utilizó el concepto de cognición distribuida como herramienta de análisis de la investigación científica. En este artículo comenzamos por explicar el origen de la noción en los ejemplos de la navegación y del procesamiento distribuido en paralelo, así como la aplicación que Giere hace al caso de la ciencia. Seguidamente, examinamos las ventajas explicativas de la cognición distribuida dentro de su perspectivismo, al permitir abordar la ciencia como proceso y erosionar la línea entre lo cognitivo y lo social, así como los límites que Giere establece para su aplicación. A continuación ubicamos la posición de Giere en relación a los debates sobre la mente extendida y las sucesivas olas del mismo, centradas bien en el principio de paridad, bien en el de complementariedad, bien en el carácter social y colectivo de lo cognitivo. Al hacerlo mostramos que algunas críticas que se le han hecho no interpretan bien su posición. Por último, reevaluamos la reticencia de Giere a extender a los sistemas distribuidos ciertas propiedades mentales, presentando dos intentos recientes de establecer las condiciones en que esta extensión es razonable.

Palabras clave: cognición distribuida; mente extendida; perspectivismo; modelos; macrocognición. 


\begin{abstract}
Ronald Giere employed the concept of distributed cognition as a tool to analyse scientific research. In this paper we begin by explaining the origin of this notion in the examples of navigation and parallel distributed processing, as well as Giere's application to science. Next, we examine the explanatory advantages of distributed cognition within his perspectivism, particularly the claim that it allows to study science as a process and to erase the divide between the cognitive and the social, and the limits that Giere establishes for its application. Then we locate Giere's position in relation to the debates about the extended mind and its successive waves, focused either on the parity principle, the complementarity principle, or the collective and social nature of cognition. In doing so, we show that some criticisms of Giere miss the point. Finally, we reevaluate Giere's reluctance to extend certain mental properties to distributed cognitive systems, presenting two recent attempts to establish conditions in which such an extension is reasonable.
\end{abstract}

Keywords: distributed cognition; extended mind; perspectivism; modelos; macrocognition.

\title{
1. Introducción
}

Dentro de las contribuciones de Ronald Giere al enfoque cognitivo de la ciencia, su última etapa se caracterizó por apelar a la noción de cognición distribuida como una herramienta de análisis (Giere, 2002a, 2002b, 2006a, 2006b, 2007; Giere y Moffat, 2003). Aunque Giere no llegó a desarrollar nunca la idea ${ }^{1}$ y se limitó a aplicar al estudio de la ciencia algunas conclusiones generales extraídas de las propuestas que surgen en aquel momento en ciencia cognitiva, es interesante examinar su planteamiento en la medida en que refleja la preocupación principal que orientó su manera de acercarse a la filosofía de la ciencia: ofrecer una caracterización que evitara los extremos de una descripción puramente formal que toma las teorías como objetos abstractos cuya estructura hay que desvelar y una descripción que toma el conocimiento científico como producto puramente de factores sociales. Adoptar el enfoque cognitivo permite a su juicio seguir poniendo el foco en la práctica pero atendiendo a la especificidad de los mecanismos productores de conocimiento y no tanto a la generalidad de los elementos sociales donde dicha práctica tiene lugar. En este contexto, la noción de cognición distribuida se presenta como una posible herramienta para difuminar la línea entre lo cognitivo y lo social.

\footnotetext{
${ }^{1}$ De hecho, los escritos de Giere al respecto se caracterizan por ser muy repetitivos, apenas ańadiendo un nuevo matiz en cada uno de esos textos a la posición que defiende. Cuando en este artículo remitamos al lector a un determinado texto como referencia de lo que estamos explicando, a menudo podrá encontrar la misma idea reproducida de manera literal en alguno de los otros escritos mencionados.
} 
En este artículo revisaremos la concepción de Giere acerca de la cognición distribuida. En primer lugar, presentaremos el origen de la noción y el uso que Giere hace de la misma. Seguidamente, examinaremos las ventajas explicativas de esta noción dentro de su perspectivismo, así como los límites que Giere establece para su aplicación. Pasaremos a ubicar la posición de Giere en relación a los debates sobre la mente extendida y las sucesivas olas del mismo, mostrando que algunas críticas que se le han hecho no interpretan bien su posición. Por último, evaluaremos la reticencia de Giere a extender a los sistemas distribuidos ciertas propiedades mentales, presentando algunos intentos recientes de establecer las condiciones en que esta extensión es razonable.

\section{Qué es la cognición distribuida}

En términos generales, un sistema cognitivo distribuido es un sistema de procesamiento de información en el que no existe un elemento centralizador (el "procesador central" que postulan los modelos computacionales clásicos) en el que descansa el papel de unificar y procesar toda la información que el sistema recibe. La idea de distribución puede realizarse de diversas maneras, en función de cuáles sean los subsistemas que se relacionan, cómo se reparten las tareas y qué comunicación existe entre los mismos. Lo característico del análisis que postula Giere es que aplica la idea de distribución a los grupos de investigación científica y que se extiende no solo a las personas que lo constituyen sino también a la instrumentación con la que operan, así como a las distintas representaciones externas con las que tienen interacciones relevantes para la investigación en cuestión. Giere (2002b) contrasta la idea de cognición distribuida con la de cognición colectiva, que involucra solamente a conjuntos de personas, no a los artefactos externos con los que interaccionan.

Para dar cuenta de su posición, Giere se apoya especialmente en dos modelos de cognición distribuida. Ambos son bastante conocidos, así que nos limitaremos a presentarlos de modo sucinto. Uno de ellos es el análisis de Hutchins (1995) del sistema de navegación de un barco. Hutchins observa que, aunque existe una jerarquía de mando en la toma de decisiones, la navegación propiamente dicha no depende de un único individuo o conjunto de individuos que procesan toda la información pertinente para el mantenimiento del rumbo. Antes bien, lo que existe es un complejo sistema de interacciones entre los tripulantes, con sus roles y capacidades diferentes, y la instrumentación, que produce representaciones de diversos parámetros que determinan la posición del barco y las acciones adecuadas a realizar en cada momento. Ninguna persona puede por si sola realizar los complejos cálculos en tiempo real necesarios para ejecutar las maniobras oportunas. La labor que cada tripulante lleva a cabo descansa en la información suministrada por otros tripulantes a partir de las lecturas de los instrumentos. La jerarquía de mando emana de un entramado social e institucional que dota de una determinada organización a las interacciones, pero que no sustituye a lo 
cognitivo sino que se funde con él. El barco, constituido por la tripulación, la instrumentación y las relaciones significativas entre ellos, es el genuino sistema cognitivo distribuido.

El segundo modelo al que Giere recurre es el paradigma del procesamiento distribuido en paralelo (PDP, Rumelhart y McClelland, 1986), popularmente conocido como enfoque conexionista de la mente. Frente a la concepción "clásica" de la mente como un sistema de procesamiento en serie de símbolos, el enfoque PDP proponía sistemas de procesamiento basados en unidades simples masivamente interconectadas. El patrón de conectividad (i. e., qué unidad es activada o inhibida por otra), así como los pesos de las conexiones (i. e., cuánta activación/inhibición se recibe) determinan el comportamiento global del sistema. Es característico de los sistemas conexionistas que los pesos no vienen dados de antemano sino que se ajustan, en función de una serie de algoritmos de aprendizaje, a partir del entrenamiento de la red con una muestra relevante del dominio de información que se encarga de procesar. Un sistema PDP no sería otra cosa que un reconocedor de patrones: es capaz de reconocer en la información que recibe una serie de pautas recurrentes (v. g., maneras de conjugar los verbos en pasado) de las que extrae generalizaciones para procesar de manera adecuada la información novedosa del mismo dominio (v. g., conjugar un verbo que no estaba en el conjunto de entrenamiento).

Según Giere, el problema que se plantea a estos sistemas es el de dar cuenta del procesamiento de símbolos en serie característicos de los sistemas simbólicos clásicos. El modo de solventar el problema sería tomando en consideración como parte del procesamiento las representaciones externas con las que el sistema se relaciona ${ }^{2}$. Un ejemplo típico es la realización de una suma por medio de la manipulación de signos escritos en un papel. Al igual que en el caso de la navegación, la lección que Giere quiere extraer es que el funcionamiento del sistema solo es comprensible si introducimos en nuestra explicación esos elementos externos -instrumentos y representaciones- con los que se dan las interacciones más significativas para realizar la tarea encomendada. Su punto de vista es que el mismo tipo de análisis puede extenderse a otros sistemas que realizan funciones inteligentes, como el constituido por un determinado grupo

\footnotetext{
${ }^{2}$ En realidad, al apoyarse en esta variedad de externismo Giere está tomando implícitamente partido por una de las interpretaciones del conexionismo, pero lo cierto es que no todos los conexionistas son partidarios de esta posición. Por ejemplo, una alternativa muy discutida es la que concibe el procesamiento en serie como una descripción de alto nivel de los procesos masivamente en paralelo que tienen lugar en la red neuronal artificial (Smolensky, 1988). En otras palabras, un sistema PDP podría funcionar como uno clásico no en virtud de su interacción con ciertas representaciones externas sino de sus propias capacidades computacionales internas. En cualquier caso, Giere no parece tener un conocimiento muy detallado de las características computacionales de los sistemas conexionistas y se limita a tomarlos como inspiración genérica de su posición.
} 
científico de investigación. También en este caso la caracterización adecuada de cómo se hace la ciencia debe explicar cómo se distribuye la información entre los investigadores y los diversos elementos externos con los que interaccionan.

Giere considera que la noción de distribución se puede aplicar tanto a la observación científica como a la teorización misma. En el caso de la observación, podemos entender las instalaciones donde se realizan los experimentos científicos como parte integral de los procesos que tienen lugar cuando se hace ciencia. Al igual que la tripulación del barco de Hutchins, los investigadores realizan su trabajo por medio de una instrumentación específica, como los ordenadores o los instrumentos de medición, que arroja diversos tipos de representaciones sobre las que se distribuye la información pertinente para determinar qué es lo que se observa. En el caso de la teorización, Giere (2006a) apunta al uso de modelos como un modo de cognición distribuida. Teorizar requiere construir modelos con los que razonar, y estos modelos no son sino representaciones externas cuyas características influyen en el tipo de procesamiento de información que caracteriza a la teorización. Giere distingue cuatro grandes tipos de modelos: los diagramas, las imágenes en general (de los cuales los diagramas serían un caso específico), los modelos físicos (v.g., una maqueta) y los modelos abstractos. Los tres primeros parecen prestarse de manera más directa a una división del trabajo análoga a la que se percibe en los sistemas PDP. Los científicos no realizan todo su trabajo "en la cabeza" sino que, al igual que las redes, los científicos cuentan con capacidades de reconocimiento de patrones ${ }^{3}$ y las explotan para construir modelos en los que dichos patrones sean más fácilmente accesibles. Así, es patente que hay diagramas que son mejores que otros y uno de los motivos es su mejor adaptación a nuestras capacidades perceptuales: un diagrama con una mala distribución de las figuras o una maqueta con una escala muy pequeña pueden ser modelos inapropiados a pesar de que contengan toda la información pertinente. Es la interacción con estos modelos la que permite que la información tenga un procesamiento adecuado.

Los modelos abstractos son los que plantean el mayor desafío, pero Giere insiste en que debemos evitar la tentación de entenderlos como modelos internos, construidos "en la mente" del investigador, el cual contaría a su vez con un sistema de representaciones simbólicas con las que realiza un tipo de razonamiento abstracto. Giere da varias razones para rechazar este enfoque. Por una parte, nos recuerda que incluso los modelos abstractos se apoyan en algún tipo de representaciones externas, como un conjunto de ecuaciones. De hecho, un modelo abstracto puede típicamente involucrar la construcción de modelos concretos como

\footnotetext{
${ }^{3}$ Giere está aquí aceptando implícitamente que los modelos conexionistas son una buena caracterización del funcionamiento cognitivo interno de las personas, pero su posición general no se ve afectada demasiado por este supuesto. Incluso si la cognición humana fuera un sistema computacional de tipo von Neumann, puede seguir manteniéndose la tesis de la necesidad de apoyar la misma en la interacción con elementos externos de diversa índole.
} 
los diagramas (observación que seguramente hay que entender en el sentido de que no existirían modelos "puramente" abstractos). En consecuencia, habría que incluir las propiedades de dichas representaciones, y de nuestra interacción con ellas, en el análisis del proceso de teorización. Por otra parte, apunta al papel decisivo de la capacidad lingüística para la creación de modelos abstractos, tales como los planes. El lenguaje puede verse también como una herramienta (en palabras de Clark, 1997, el artefacto definitivo) con la que construir representaciones públicas que permiten distribuir la información entre un colectivo amplio con acceso a las mismas.

\section{Ventajas y límites de la concepción distribuida de la ciencia}

Giere se enfrenta a la pregunta de qué es lo que aporta este enfoque distribuido de la ciencia al análisis de la misma. ¿¿No se estaría en el fondo repitiendo, con otros términos, afirmaciones conocidas, y un tanto triviales, acerca del carácter colectivo de la práctica científica? Giere reivindica que el recurso a la cognición distribuida supone una aportación novedosa. Por una parte, subraya que la idea de distribución pone el foco en el proceso de adquisición del conocimiento y no en la posesión estática del mismo (Giere, 2002a). La idea no es sustituir la concepción del conocimiento como algo depositado en un individuo por una concepción en la que el conocimiento se encuentra depositado en un conjunto de individuos, instrumentos y representaciones. Lo que la idea de distribución pretende captar son las interacciones entre esos tres diferentes elementos, de manera que las variaciones en las mismas suponen una variación del proceso cognitivo en cuestión. Giere toma de Herbert Simon la idea de que puede haber representaciones (v.g., un conjunto de proposiciones y un diagrama) que sean equivalentes informacionalmente pero no computacionalmente: el modo de manipular unas y otras es diferente $y$, en consecuencia, también lo es el proceso cognitivo distribuido en el que participan.

Por otra parte, la idea de la cognición distribuida permite librarse del supuesto de que existe una división drástica entre lo cognitivo y lo social. Frente a la concepción constructivista de que la formación de las creencias científicas obedece a factores puramente sociales, la idea de distribución permite ver estos factores como parte de un sistema cognitivo y, en consecuencia, aplicar la ciencia cognitiva al estudio de la ciencia en general. Giere y Moffat (2003) critican la idea de Latour y Woolgar (1986) de presentar lo cognitivo y lo social en una oposición binaria, con lo social ejerciendo el papel dominante. La cognición distribuida permite ver cómo lo cognitivo y lo social se solapan, como en el complejo de relaciones institucionales y de interacciones a bordo del barco descritas en el ejemplo de Hutchins.

Rechazar el constructivismo no implica que el conocimiento de los sujetos implicados sea una compilación de creencias atemporales, sino que puede verse 
como un conjunto de fragmentos individuales de información que se encuentran coordinados en un sistema organizado que opera en tiempo real. De este modo, se da un refuerzo mutuo entre la idea de distribución y el perspectivismo que constituye su propuesta epistemológica (Giere, 2006a). El perspectivismo se opone tanto al realismo objetivista, que considera que las afirmaciones científicas son descripciones objetivas de una realidad independiente, como al relativismo construccionista, que las considera producto de la sociedad y tan variables como esta. Para Giere cada sujeto epistémico accede a una perspectiva parcial de la realidad, pero esto no degenera en relativismo. Así, las distintas perspectivas visuales de algo que contemplamos poseen objetividad intersubjetiva. Como en el caso de los colores, podemos comprometernos con la realidad de lo percibido y al mismo tiempo aceptar que dicha realidad depende de la perspectiva, v.g., del funcionamiento de nuestros órganos visuales en interacción con la iluminación del entorno.

Giere pretende extender el perspectivismo a la observación y teorización científica. Es aquí donde la idea de cognición distribuida desempeña su papel. La observación depende crucialmente del uso de la instrumentación, que es tan perspectivista como el de la visión: al igual que los órganos visuales, los instrumentos poseen cierta constitución interna y son sensibles a ciertos inputs y no otros. El avance de la ciencia no puede considerarse al margen de la creación de nuevos instrumentos, que reorganizan el proceso cognitivo y la distribución de tareas. El perspectivismo se extiende además a la teorización: existe una perspectiva de la mecánica clásica, otra de la electromagnética, de la cuántica, etc. Estas perspectivas plantean principios generales pero no hacen afirmaciones sobre el mundo: son los modelos los que las hacen. Estos, como hemos visto, son representaciones externas que podemos manipular y estas manipulaciones forman parte de los procesos cognitivos que producen el conocimiento. Los modelos, por otra parte, se aplican solamente a ciertos aspectos del mundo y su precisión puede ser más detallada o más general. Este realismo perspectivista, sostiene Giere, es el máximo que se puede conseguir; el realismo objetivista no es ni siquiera un ideal.

No obstante, para Giere la idea de distribución no debe utilizarse de manera irrestricta. En particular, rechaza los usos de la misma para redefinir los límites de la mente. Giere (2007) revisa el famoso ejemplo de Clark y Chalmers $(1998)^{4} \mathrm{de}$ Otto, una persona con Alzheimer que utiliza una libreta como memoria externa. Aunque comparte la conclusión de que esta especial relación entre sujeto y artefacto permite entenderlos como un sistema cognitivo distribuido entre ambos, rechaza que la mente de Otto se extienda hasta la libreta o que las anotaciones de la misma constituyan literalmente sus creencias. La concepción de un agente

\footnotetext{
${ }^{4}$ La fuente de Giere es en realidad el libro de Clark (1997). Por vicisitudes editoriales, el artículo fundacional de Clark y Chalmers sobre la mente extendida no fue publicado hasta 1998 aunque circulaba ampliamente antes de esa fecha. Por otra parte, de los dos autores originales es Clark quien desarrolló posteriormente la tesis y es con él con quien típicamente se asocia.
} 
humano, con intencionalidad, consciencia o creencias, forma parte de nuestra cultura y desempeńa un papel explicativo cotidiano. Extenderla a los artefactos o a los grupos de investigación acarrea más confusión que ganancias explicativas. Dańar la libreta de Otto es moralmente reprobable pero no supone una alteración de la mente de Otto, sino de los procesos cognitivos que tienen lugar en el sistema localmente distribuido que forman el sujeto y su libreta.

A diferencia de la idea de mente, Giere considera que la idea de cognición es más técnica: una herramienta de análisis teórico que nos es de utilidad para caracterizar el funcionamiento de ciertas entidades. El empleo de estas nociones teóricas solo está constreñido por los dividendos explicativos que arroje: podemos recurrir a la caracterización de la ciencia como un sistema distribuido en la medida en que esto nos permita ofrecer explicaciones fuera del alcance de un análisis puramente individualista o puramente social. Por el contrario, intentar redefinir los límites de lo mental sobre la misma base obligaría al mismo tiempo a redefinir todas esas nociones cotidianas en las que la mente está implicada. El intento parece abocado al fracaso dado que carecemos de criterios para determinar dónde debe detenerse la búsqueda.

\section{Extender lo cognitivo: paridad, complementariedad y la relación indivi- dual/social}

Como ya hemos dicho, Giere solo toma la idea de cognición distribuida como marco general y no se adentra en los debates sobre la misma que tienen lugar en ciencia cognitiva. No obstante, para comprender mejor su propuesta resulta pertinente examinarla en relación a los hitos principales de dicho debate. Se pueden distinguir tres sucesivas olas en el desarrollo de la tesis de la mente extendida (Sutton, 2010). La primera, representada por el trabajo fundacional de Clark y Chalmers, apoya su argumentación en el principio de paridad, que supone una extensión de la equivalencia funcional fuera de las fronteras del cerebro. La idea de fondo es que si un fenómeno localizado dentro del cerebro y otro fuera de la mente son funcionalmente análogos, entonces pueden considerarse equivalentes en tanto fenómenos cognitivos. La segunda ola descansa en el principio de complementariedad y ofrece una perspectiva integracionista de la cognición. Los fenómenos internos y externos pueden ser radicalmente diferentes pero pueden considerarse parte del mismo sistema cognitivo en la medida en que son complementarios para la realización de una tarea cognitiva. Por ejemplo, Wilson (2010) propone considerar los recursos cognitivos del mismo modo en que la biología evolucionista considera los recursos para el desarrollo. Cualquier criatura depende de su legado genético para sobrevivir, pero su adaptación también está condicionada por los recursos ambientales que puede usar para mejorar su aptitud biológica. De la misma manera, las criaturas pueden encontrar en el entorno recursos que mejoran sus capacidades cognitivas. La mayoría de nuestras tareas cognitivas dependen de la integración causal entre estas estructuras del ambiente 
y nuestras capacidades heredadas. Esta integración produce un proceso cognitivo genuinamente nuevo y diferente de los procesos internos de los individuos. La tercera ola supone un cambio desde la perspectiva individualista a la perspectiva grupal. El acento se pone ahora en la dimensión social de la cognición y en entender la mente como una propiedad de los colectivos. Esta tercera ola evita la espinosa cuestión de los límites de la cognición (Cash, 2013). No es necesario preguntarse hasta dónde se extiende la mente de Otto o de cualquier otro sujeto, porque lo cognitivo abarca la comunidad y podemos percibir en ellas propiedades tales como agencia colectiva, creencias colectivas o incluso personas colectivas (Huebner, 2014; Theiner et al., 2010). Esto no supone negar la existencia de dinámicas cognitivas individuales sino concebirlas como fuertemente influenciadas y parcialmente constituidas por ciertas estructuras sociales que caracterizan nuestro entorno público, particularmente las instituciones, las normas sociales y las prácticas culturales. Estas proporcionan esquemas conceptuales externos que no existen en las mentes individuales sino como estructuras compartidas que constituyen auténticas instituciones mentales (Gallagher, 2013).

Por el momento en que escribe y por los autores en los que se basa, podría pensarse que Giere razona desde principios análogos a los de la primera ola, particularmente el de paridad. Creemos que esto solo es en parte así. Es cierto que Giere basa partes de su razonamiento en una analogía funcional entre procesos internos y externos, v.g., al comparar el papel de los instrumentos de registro con el de los órganos perceptuales. Sin embargo, dado que su empleo del término 'cognición' es técnico y pragmático, el cometido de estas analogías no es establecer qué forma parte de la ontología cognitiva, sino únicamente dilucidar el papel que desempeña cada elemento en la investigación científica que, como sistema productor de conocimiento, puede caracterizarse de suyo como cognitiva. Pero también es cierto que pueden percibirse en su visión elementos que caracterizan tanto a la segunda como a la tercera ola. En primer lugar, para Giere personas, instrumentos y representaciones externas se complementan aunque mantienen diferentes propiedades y dinámicas de interacción. Por ejemplo, tenga o no tenga algún carácter computacional la cognición humana, la manipulación de modelos físicos claramente no lo tiene (Giere, 2006a). En segundo lugar, como ya hemos dicho, para Giere la noción de distribución permite obliterar la línea entre lo cognitivo y lo social. Tal vez la diferencia principal con los postulados de la tercera ola resida en que esta última otorga a lo social un papel constitutivo más fuerte mientras que para Giere la pregunta sobre qué constituye qué resultaría más bien ociosa. Pero esto no debe ocultar la coincidencia fundamental en la idea de incluir dentro del análisis cognitivo factores procedentes del sistema de relaciones sociales y de las interacciones de estas con el entorno.

Teniendo esto en cuenta, no están del todo justificadas las críticas que se han vertido sobre Giere reprochándole que su concepción de la cognición distribuida sigue siendo individualista (Vaesen, 2011; Brown, 2015). Es cierto que Giere no rechaza aplicar la noción de cognición a lo que ocurre en la maquinaria interna 
de los individuos; de hecho, nunca está en cuestión que estos constituyen subsistemas cognitivos por sí mismos. Pero esto no significa que su posición sea individualista. Esto se puede percibir igualmente en sus consideraciones críticas sobre el análisis de Knorr-Cetina (1999) de la cultura epistémica de la física de altas energías. Los proyectos en este ámbito involucran a cientos de científicos en instalaciones que son como pequeńas ciudades. Allí la producción del conocimiento no es atribuible a un científico en particular, ni siquiera a un grupo de ellos o a un determinado laboratorio. Más bien, sostiene esta autora, el conocimiento lo producen los experimentos mismos. En consonancia con esto, ella sugiere que es posible atribuir a los propios experimentos un tipo de "autoconocimiento" generado por los procedimientos realizados y el intercambio de información.

La propuesta de Knorr-Cetina, argumenta Giere (2002b), es consecuencia de la insistencia en buscar un sujeto epistémico. Al no encontrarlo en los individuos, se ve forzada a ubicarlo en otra parte y no encuentra una unidad menor que el experimento. Pero del hecho de que se produzca conocimiento, señala él, no hay por qué deducir que hay un sujeto produciéndolo. Podemos tener cognición distribuida sin necesidad de hablar de conocimiento distribuido (Giere, 2007). Cada individuo tiene el conocimiento que tiene, desde su perspectiva particular, que nunca es exactamente la misma que la de otro individuo. En realidad, buena parte del conocimiento científico puede formularse de un modo impersonal, con expresiones como "Este experimento muestra que... / lleva a la conclusión de que...". Aquellos que sacan las conclusiones en cuestión sí son individuos: en primer lugar los científicos y en segundo la audiencia a la que llegan. Pero este no es un tipo de individualismo que concierna a la cognición distribuida: los individuos en cuestión no desempeñan un papel privilegiado en el proceso cognitivo o en la producción del conocimiento. Sus "privilegios" se circunscriben al hecho de que es a ellos a quienes hay que atribuir agencia, creencias o consciencia. Nótese que ni siquiera la tercera ola de la mente extendida rechaza estas atribuciones individuales; el énfasis es más bien en que es posible realizar además atribuciones colectivas de propiedades análogas y que estas gozan de una cierta prioridad sobre las individuales, en la medida en que estas últimas dependen de una normatividad que emana de lo social.

\section{5. ¿Existen condiciones para una mente colectiva?}

Ya hemos dicho que para Giere la noción de cognición tiene un carácter técnico y teórico, de manera que su empleo está supeditado a la capacidad de proporcionar una explicación de determinados procesos. La cuestión de cuándo un sistema sea "genuinamente cognitivo" no se plantea para él. En realidad, el hecho de que su objeto de análisis sean los sistemas que producen conocimiento científico permite que tal cuestión sea obviada: estos sistemas necesariamente involucran seres humanos. En consecuencia, las sucesivas olas de la extensión de la mente parecen moverse en la dirección que él precisamente quiere evitar. Ahora 
bien, nos preguntamos, ¿¿debe evitarla? Creemos que no. La distinción entre 'cognitivo', como término técnico, y 'mental', como cotidiano y cultural, se antoja un tanto arbitraria, habida cuenta que lo primero precisamente se utiliza para fundamentar ciertas características de lo segundo. Si lo que se quiere es argumentar contra una extensión al colectivo de ciertas propiedades mentales típicas de los individuos debe hacerse de manera fundamentada y no por apelación a los usos cotidianos de los términos. Y lo cierto es que es un terreno de estudio fértil el que indaga las condiciones en que dicha extensión puede tener lugar. Examinemos dos propuestas representativas al respecto.

Una de ellas es el análisis de la macrocognición propuesto por Huebner (2014), quien cree que es posible encontrar criterios que determinen cuándo nos encontramos con una mente colectiva y no con un mero sistema de información distribuida. La clave se encontraría en la capacidad de un sujeto colectivo para producir y consumir determinadas representaciones de un modo que no es reducible a sus integrantes individuales. Por ejemplo, un equipo organizado en diferentes subsistemas, cada uno de los cuales analiza diferentes elementos del entorno y genera la información relativa al mismo, contaría como una mente colectiva si y solo si no existe un sistema central de toma de decisiones que recolecta, procesa y utiliza toda la información recopilada por los diversos subsistemas. Un genuino agente colectivo debe cumplir con el requisito de que estén distribuidas tanto la recolección como el almacenamiento y uso de las representaciones. Este requisito se puede cumplir en diversos grados. En un extremo podemos encontrarnos con mentes colectivas mínimas, como la que constituye una colonia de abejas, y en el otro con mentes colectivas máximas, como la Organización Europea para la Investigación Nuclear (CERN). En el CERN los distintos subsistemas reciben actualizaciones de lo que ocurre en el resto de la estructura a través de informes internos, memorandos, correos, etc. La información se recoge y evalúa localmente y el resto del personal recibe noticia de ella en informes internos cortos, que actúan como representaciones públicamente compartidas y que persisten durante un largo tiempo como representaciones externas que permiten guiar la investigación en el curso de los años.

Un segundo ejemplo lo encontramos en Theiner et al. (2010), quienes defienden también la posibilidad de una mente grupal y exploran las condiciones en que esta puede tener lugar. La cognición en el grupo no puede ser simplemente un agregado desestructurado de cogniciones individuales sino que tiene que ser el resultado de una división clara del trabajo entre los miembros del grupo, lo cual implica la especialización cognitiva. Un ejemplo representativo de mentalidad colectiva se encuentra en la memoria transactiva: lo que hay que recordar se encuentra repartido entre los distintos miembros del colectivo, quienes cuentan además con una meta-memoria que les permite determinar "quién recuerda qué" y así saber dónde dirigirse para recuperar la información requerida. Pero esto no significa que podamos extender al colectivo todo tipo de propiedades mentales que atribuimos a los componentes cognitivos individuales. Del mismo modo 
que no atribuimos al nivel neuronal de un sujeto las mismas propiedades que le atribuimos cuando lo consideramos en tanto agente personal (v. g., no atribuimos creencias a las neuronas) sería ocioso atribuir al nivel colectivo todo tipo de propiedades del nivel personal, como sería hablar, por ejemplo, de consciencia colectiva. Ahora bien, ¿por qué sí atribuir memoria y no consciencia? Theiner $e t$ al. proponen tres condiciones para atribuir propiedades cognitivas al colectivo: primero, el sistema tiene que estar integrado y producir ganancias funcionales con respecto a la actuación por separado de sus integrantes; segundo, el grupo produce efectos sistémicos al operar como una única totalidad causal; tercero, la ganancia funcional es posible cuando el sistema mejora las capacidades individuales de sus constituyentes o manifiesta nuevas habilidades emergentes. Estas condiciones se cumplirían en el caso de la memoria: volviendo a casos como el CERN, hay creencias que el sistema posee aunque ninguno de sus componentes por separado las posea. Estas creencias pertenecen a todos en la medida en que existan procedimientos fiables que permitan acceder a ellas. Pero las condiciones no se cumplirían respecto a la consciencia: no se ve ningún sentido en que podamos hablar de una consciencia que pertenece a todos; no hay propiedades conscientes más allá de las de los individuos.

Sin entrar a evaluar las virtudes y defectos de estas propuestas, lo que muestran es que hay vías de tratamiento para la preocupación de Giere de no sobrepasarse en la aplicación de propiedades mentales a las entidades involucradas en la práctica científica. Estos tratamientos parecen aplicarse a buena parte de las propiedades causales-funcionales, como la memoria, la intencionalidad o incluso la agencialidad. Las que quedan al margen son las propiedades con rasgos fenoménicos, como la siempre peliaguda consciencia.

\section{Conclusión}

La idea de cognición distribuida funciona como una suerte de interfaz entre la cognición individual y la social, pero Giere nunca llegó a ofrecer un desarrollo adecuado de esta tesis. Este es quizás un problema común de algunas de las intuiciones, sugerentes pero poco elaboradas, ofrecidas por los pioneros en el enfoque cognitivo de la ciencia, lo que los convierte en diana fácil de los críticos con este enfoque (v. g., Glymour, 1992). En este artículo hemos pretendido mostrar cómo los desarrollos y debates posteriores en ciencia cognitiva en torno a la idea de distribución permiten refinarla, así como evaluar de un modo más razonado las perspectivas explicativas que ofrece dicha noción para el análisis de la ciencia. 


\section{Agradecimientos}

Este trabajo ha sido financiado por la Agencia Estatal de Investigación, en el marco del Proyecto de Investigación de referencia PID2019-108870GB-I00 / AEI /10.13039/501100011033, Ministerio de Ciencia e Innovación. El artículo es completamente colaborativo y el orden de autoría es alfabético.

\section{Referencias bibliográficas}

Brown, Matthew J. (2015). A Critical Appreciation of Ronald N. Giere's "Distributed Cognition without Distributed Knowing". Social Epistemology Review and Reply Collective, 4(6), 45-51.

Cash, Mason (2013). Cognition without Borders : "Third Wave" Socially Distributed Cognition and Relational Autonomy. Cognitive Systems Research, 25-26, 61-71.

Clark, Andy (1997). Being There: Putting Brain, Body, and World Together Again. Cambridge, MA: MIT Press.

Clark, Andy y Chalmers, David (1998). The Extended Mind. Analysis, 58(1), 7-19.

Gallagher, Shaun (2013). The Socially Extended Mind. Cognitive Systems Research, 2526, 4-12.

Giere, Ronald N. (2002a). Scientific Cognition as Distributed Cognition. En Peter Carruthers, Stephen Stitch y Michael Siegal (eds.), The Cognitive Basis of Science (pp. 285-299). Cambridge: Cambridge University Press.

Giere, Ronald N. (2002b). Distributed Cognition in Epistemic Cultures. Philosophy of Science, 69, 637-644.

Giere, Ronald N., Moffat, Barton (2003). Distributed Cognition: Where the Cognitive and the Social Merge. Social Studies of Science, 33(2), 301-310.

Giere, Ronald N. (2006a). Scientific Perspectivism. Chicago: University of Chicago Press.

Giere, Ronald N. (2006b). The Role of Agency in Distributed Cognitive Systems. Philosophy of Science, 73(5), 710-719.

Giere, Ronald N. (2007). Distributed Cognition without Distributed Knowing. Social Epistemology, 21(3), 313-320.

Glymour, Clark (1992). Invasion of the Mind Snatchers. En R. Giere (ed.), Cognitive Models of Science (Minnesota Studies in the Philosophy of Science) (pp. 465-471). Minnesota: University of Minnesota Press.

Huebner, Bryce (2014). Macrocognition: A Theory of Distributed Minds and Collective Intentionality. New York: Oxford University Press.

Hutchins, Edwin (1995). Cognition in the Wild. Cambridge, MA: MIT Press.

Knorr-Cetina, Karin (1999). Epistemic Cultures: How the Sciences Make Knowledge. Cambridge, MA: Harvard University Press. 
Latour, Bruno y Woolgar, Steve (1986). Laboratory Life: The Construction of Scientific Facts. Princeton, NJ: Princeton University Press.

Rumelhart, David E., McClelland, James L., y PDP Research Group (1986). Parallel Distributed Processing: Explorations of the Microstructure of Cognition (Vols. 1 y 2). Cambridge, MA: MIT Press.

Smolensky, Paul (1988). On the Proper Treatment of Connectionism. Behavioral and Brain Sciences, 11, 1-74.

Sutton, John (2010). Exograms and Interdisciplinarity: History, the Extended Mind, and the Civilizing Process. En Richard Menary (ed.), The Extended Mind (pp. 189-225). Cambridge, MA: MIT Press.

Theiner, Georg, Allen, Colin, y Goldstone, Robert L. (2010). Recognizing Group Cognition. Cognitive Systems Research, 11(4), 378-395.

Vaesen, Krist (2011). Giere's (In)Appropriation of Distributed Cognition. Social Epistemology, 25(4), 379-391.

Wilson, Robert A. (2010). Meaning Making and the Mind of the Externalist. En Richard Menary (ed.), The Extended Mind (pp. 167-188). Cambridge, MA: MIT Press. 